\title{
Influence of vegetation structure, seasonality, and soil physical properties on rodent species diversity and community assemblages on West Mount Kilimanjaro, Tanzania.
}

\author{
SUZANA THOMAS ${ }^{1}$, LOTH MULUNGU $^{2}$, and GEOFREY SOKA ${ }^{1}$ \\ ${ }^{1}$ Sokoine University of Agriculture College of Forestry Wildlife and Tourism \\ ${ }^{2}$ Sokoine University of Agriculture College of Veterinary Medicine and Biomedical Sciences
}

December 2, 2021

\begin{abstract}
A study on rodent species diversity and community assemblages in West Mt Kilimanjaro was conducted in seven different habitats, covering two dry and wet seasons. Data were collected using a combination of medium-sized Sherman's live traps, snap and Havarhart traps, for three consecutive nights. General Linear Models (GLM) were used to analyze the effects of predictors (vegetation attributes, seasonality, soil physical properties, disturbance and altitude) on rodent species richness and abundance. Community structure analysis was conducted in the Primer v6 program and Canonical correspondence analysis for habitat association in PAST. A total of 1,393 individuals from 14 species of rodents were trapped. The most dominant rodent species were Rhabdomys pumilioPraomys delectorum, and Lophuromys verhageni which contributed to $68.86 \%$ of the total captures. Lophuromys verhageni occurred across all the habitats and seasons. Moreover, habitat types, seasonality, soil texture, ground cover, and altitude significantly influenced rodent species abundance $(\mathrm{P}<0.05)$. Furthermore, habitat types, seasonality and altitude significantly influenced rodent species richness $(\mathrm{F} 8,759=629.7, \mathrm{p}<0.001, \mathrm{R} 2=0.87)$. In addition to that, two major rodent communities were formed in different habitats. The results show that rodent species richness, abundance, and community assemblages in Mt Kilimanjaro, are a result of change in vegetation structure along the altitudinal gradients. Therefore, information on habitat requirements of multiple species is crucial for the management and conservation of these communities.
\end{abstract}

\section{Hosted file}

Manuscript Main Dcument.doc available at https://authorea.com/users/449120/articles/547718influence-of-vegetation-structure-seasonality-and-soil-physical-properties-on-rodentspecies-diversity-and-community-assemblages-on-west-mount-kilimanjaro-tanzania 

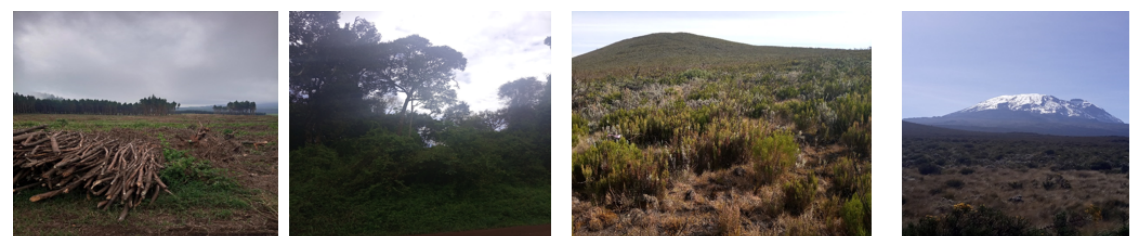

Vegetation type and structure changes along the altitudinal gradient
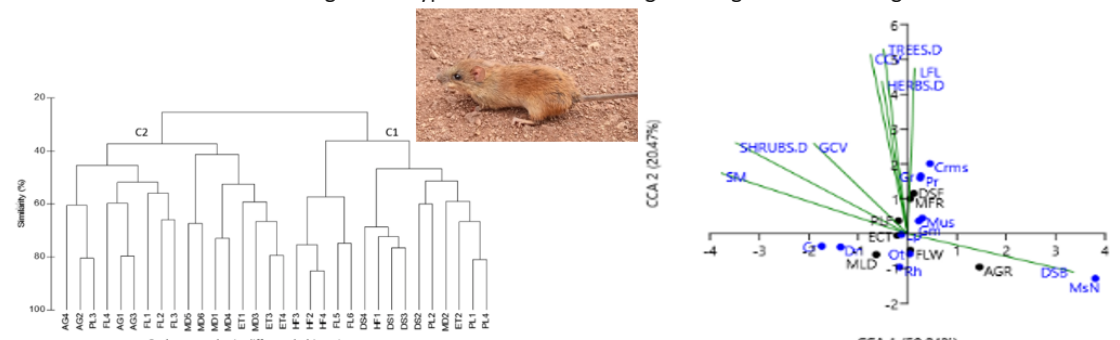

CCA 1 (59.04\%) 